\title{
Role of filament plasma remnants in ICMEs leading to geomagnetic storms
}

\author{
Rahul Sharma ${ }^{1}$, Nandita Srivastava ${ }^{2}$ and D. Chakrabarty ${ }^{3}$ \\ ${ }^{1} 3$, Indra Nagar, North Sunderwas, Udaipur, India. \\ email: sharmarahul20@googlemail.com \\ ${ }^{2}$ Udaipur Solar Observatory, Physical Research Laboratory, Udaipur, India. \\ email: nandita@prl.res.in \\ ${ }^{3}$ Space and Atmospheric Sciences Division, Physical Research Laboratory, Ahmedabad, India. \\ email: dipu@prl.res.in
}

\begin{abstract}
We studied three interplanetary coronal mass ejections associated with solar eruptive filaments. Filament plasma remnants embedded in these structures were identified using plasma, magnetic and compositional signatures. These features when impacted the Earth's terrestrial magnetosphere - ionosphere system, resulted in geomagnetic storms. During the main phase of associated storms, along with high density plasma structures, polarity reversals in the Y-component (dawn-to-dusk) of the interplanetary electric field seem to trigger major auroral substorms with concomitant changes in the polar ionospheric electric field. Here, we examine the cases where plasma dynamics and magnetic structuring in the presence of the prompt penetration of the electric field into the equatorial ionosphere affected the space weather while highlighting the complex geomagnetic storm-substorm relationship.
\end{abstract}

Keywords. Solar filaments, ICMEs, geomagnetic storms, substorms

\section{Introduction}

Nearly $70 \%$ of Coronal Mass Ejections (CMEs) are associated with erupting filaments (Webb \& Hundhausen, 1987) and identification of source region remnants are rare in spacecraft measurements. Low- $\beta$ magneto-plasmas injected into the solar wind during these energetic events plays an important role in causing geomagnetic storms. While the geomagnetic storms are externally driven by solar wind drivers, the magnetospheric substorms involve a sequence of processes in the Earth's magnetosphere during which energy is extracted from the solar wind and deposited in the magnetosphere and the ionosphere (McPherron, 1979). To understand the magnetospheric substorm it is necessary to determine whether substorm onset is always externally triggered by the interplanetary sources or it occurs spontaneously as a result of internal processes. Indicators for a near-Earth location of the substorm onset are poorly understood and the relationship between the magnetic storm and substorm is controversial (Kamide et al., 1998). The research presented here investigates the role of solar filament material in triggering the substorms in the main/recovery phase of 3 geomagnetic storms related to CMEs associated with eruptive filaments.

\section{Data and Analysis}

The three reported interplanetary CME events associated with eruptive filaments arrived at $1 \mathrm{AU}$ on 21 Feb 2000, 21 Oct 2001 and 7 Jan 2005. The in - situ properties were investigated through magnetic (MFI/Wind, MAG/ACE) and plasma (3DP/Wind, 
SWE/Wind, SWEPAM/ACE and SWICS/ACE) instruments onboard the ACE and Wind spacecraft. The geomagnetic response has been studied using indices obtained from the NASA/GSFC CDAWeb (www.cdaweb.gsfc.nasa.gov/istp_public/). Filament plasma were identified using signatures described in Sharma \& Srivastava (2012), while the resulting geomagnetic effects of events are studied by a combination of interplanetary and geomagnetic indices as reported by Sharma et al. (2013).

\section{Results and Conclusion}

21 Feb 2000 event: The interplanetary CME arrived at 1 AU with a shock at 9:50 UT on 20 Feb, followed by a magnetic cloud at 5:37 UT on 21 Feb. Filament plasma located over magnetic flux rope structure arrived during the interval 16:13 UT - 19:17 UT on 21 Feb, preceded by a low temperature $\left(1.8 \times 10^{4} \mathrm{~K}\right)$ and high density $(31 \mathrm{n} / \mathrm{cc})$ pressure pulse at 15:00 UT. The cloud had a Dst index of -26 nT along with two substorms (A1 and A2) associated with the pressure pulse and later with filament material. The former (A1) is observed with sharp polarity changes in Z- component of interplanetary magnetic field (IMF Bz) and the Y- component interplanetary electric field (IEFy), while the changes in these parameters are accompanied with increase in ram pressure for A2 which is correlated with the filament remnant.

21 Oct 2001 event: The magnetic cloud is observed during the interval from 19:23 UT on 21 Oct to 00:47 UT on 22 Oct with a high density $(65 \mathrm{n} / \mathrm{cc})$ structure with temperature of the order of $10^{5} \mathrm{~K}$ at the rear boundary of the cloud with depressed ionic thermal velocities, RMS fluctuations, charge states and elevated $\alpha / p$ ratio. Upon comparing the arrival time of this structure (23:15 UT on 21 Oct) to the sudden impulse in AL and PC indices (23:21 UT on 21 Oct), we found insignificant propagation delay $(\approx 6 \mathrm{~min})$ of interplanetary feature to the magnetosphere, highlighting the potential role of high density plasma in triggering mechanisms of substorms.

7 Jan 2005 event: Two interacting magnetic clouds (MC1 and MC2) associated with two distinct erupted filaments arrived at $1 \mathrm{AU}$ and resulted into three (C1, C2 and C3) significantly sharp polarity changes $(\mathrm{C} 1 \approx 12: 00 \mathrm{UT}, \mathrm{C} 2 \approx 22: 00 \mathrm{UT}$ on 7 Jan and $\mathrm{C} 3 \approx$ 07:00 UT on 8 Jan) in IMF Bz (southward transitions) and IEFy (eastward transitions) during 7-9 January 2005. C1 and C2 brought in proportionate changes in the PC index and AL while the effect of $\mathrm{C} 3$ on PC index was disproportionately small and its effect on AL was absent. Upon comparison of the arrival times of MC1 and MC2 at 1 AU with the development of the main phase of the storm and triggering of substorms, it is clear that MC2 has almost negligible impact on the terrestrial magnetosphere-ionosphere system.

This study concludes that there is a possibility that filament plasma remnants in interplanetary CMEs can contribute to the triggering of substorms. Out of the three reported cases, two (Feb, 2000 and Oct, 2001) suggest triggering through compression by the filament plasma and further a "cause - result" relationship. The third (Jan, 2005) event highlights the role of magnetic cloud interactions and that the triggering might be caused or enhanced by discontinuities at the interface region between two clouds.

\section{References}

Kamide, Y., et al. 1998, J. Geophys. Res., 103, 17705

McPherron, R. L. 1979, Rev. Geophys. Space Phys., 17(4), 651

Sharma, R. \& Srivastava, N. 2012, J. Space Weather Space Clim., 2, A10

Sharma, R., Srivastava, N., Chakrabarty, D., Möstl, C., \& Hu, Q. 2013, J. Geophys. Res., 118

Webb, D. F. \& Hundhausen, A. J. 1987, Sol. Phys., 108, 383 